\title{
Cascading indirect genetic effects in a clonal vertebrate
}

3 Amber M. Makowicz ${ }^{* 1}$, David Bierbach ${ }^{2,3,4}$, Christian Richardson $^{1}$, Kimberly A. Hughes $^{1}$

${ }^{1}$ Department of Biological Sciences, Florida State University, 319 Stadium Drive, Tallahassee, FL 32304

${ }^{2}$ Department of Biology and Ecology of Fishes, Leibniz Institute of Freshwater Ecology and Inland Fisheries, Müggelseedamm

310, 12587 Berlin, Germany

${ }^{3}$ Excellence Cluster 'Science of Intelligence,' Technische Universität Berlin, Berlin, Germany

${ }^{4}$ Faculty of Life Sciences, Thaer-Institute, Humboldt-Universität zu Berlin, Berlin, Germany

11 Short title: Cascading IGE in Amazon mollies

13 Keywords: cascading IGE, exploratory behaviors, gene x environment interactions, gynogens,

14 Poecilia formosa, stress behaviors

\section{Author contributions}

17 This study was conceived by AMM and KAH, data collected by AMM and CR, analyzed the

18 data by $\mathrm{KAH}, \mathrm{AMM}$ and DB, and the manuscript was written by AMM and KAH.

\section{Data archiving}

21 All data will be archived in Figshare upon acceptance.

\section{Abstract}

Understanding how individual differences among organisms arise and how their effects propagate through social groups are fundamental questions in behavioral biology. Genetic

26 variation among social partners can influence individual phenotypes, creating individual

27 differences that might then have cascading effects in social groups. Using a clonal species, the 
28 Amazon molly (Poecilia formosa), we test the hypothesis that such indirect genetic effects (IGE)

29 propagate beyond individuals that experience them firsthand. We tested this hypothesis by

30 exposing genetically identical Amazon mollies to social partners of different genotypes, and

31 then moving these individuals to new social groups in which they were the only member to

32 have experienced the IGE. We found that the differences in aggression experienced in

33 genetically different social environments carried over into new social groups to influence the

34 exploratory behaviors of individuals that did not directly experience the previous social

35 environments. Our data reveal that IGE can propagate beyond the individuals that directly

36 experience them in Amazon mollies and possibly in many group-living species. Theoretical and

37 empirical expansion of the quantitative genetic framework developed for IGE to include

cascading and other types of carry-over effects will facilitate understanding of among-

39 individual variation, social behavior and its evolution.

\section{Introduction}

Interactions among individuals define the social environment and individual differences

43 have long been known to influence these interactions [1, 2]. Understanding how individual

44 differences arise and how their effects propagate through social groups are fundamental

45 questions in behavioral biology. One cause of both individual variation and propagation of

46 effects in social groups are indirect genetic effects (IGE) [3-5]. IGE arise when an individual's

47 phenotype is influenced by the genotype of its social partners, and they have been documented

48 to affect behavioral, life history, and morphological traits in a wide variety of taxa [e.g., 6-16].

49 Most of the IGE literature focuses on how stimulus genotypes influence the phenotype of focal

50 individuals. While understanding these dyadic interactions is important, much less is known

51 about IGE on group-level characteristics or the degree to which IGE can propagate to affect

52 phenotypes of individuals that do not experience them firsthand. Because IGE can profoundly

53 affect phenotypes, fitness, and the rate and direction of evolutionary change [17-19], 
54 understanding possible cascading or carry-over effects within social groups is necessary to understand behavioral variation and evolution.

There have been two studies, to our knowledge, that have investigated IGE beyond

57 those caused by dyadic interactions. The first used fruit flies (Drosophila melanogaster), to

58 measure first-order IGE on male aggressive behavior (i.e., how the genotype of stimulus

59 individuals influences the phenotypes of individuals with which they interact) and second-

60 order IGE (i.e., effects of the stimulus genotypes on the interaction between two other members

61 of the group) [20]. This experiment showed that the stimulus genotypes differed in their first-

62 order effects on individuals, and on second-order effects on interactions between other group

63 members. The second study, also using D. melanogaster, reported that the genotype of stimulus

64 individuals influenced emergent, group-level behavior of focal individuals [21]. Specifically,

65 these investigators reported that, if individuals of the stimulus genotype were more cohesive

66 (i.e., closer to one another, on average), then the focal individuals were also more cohesive, and

67 interactions between stimulus and focal individuals were less frequent.

of one individual on another. However, it remains unknown whether IGE previously

experienced by one or a few group members can influence the behavior of individuals that were never exposed to the IGE. That is, can IGE propagate beyond individuals that experience them

firsthand? Previous work indicates that individual group members can influence group behavior [1, 22-26]. However, this literature has generally not focused on prior social experience as a factor that generates differences between influential group members [but see 27, 28], and we know of no studies that implicate IGE as a cause of such differences. Because many organisms exhibit either dispersal or fission-fusion social structure, understanding IGE caused

77 by prior social environments is critical to understanding the evolution and ecology of collective

78 behaviors. Furthermore, it is challenging to measure prior influence of IGE because it is difficult

79 to replicate group genotypic composition and genetically-based differences in social experience in sexually-reproducing species. 
Naturally clonally-reproducing organisms provide an opportunity to measure these

effects outside of model species and without inbreeding or complex breeding designs. The Amazon molly (Poecilia formosa) is a gynogenetic, all-female species [29] that arose from a single hybridization event between a male sailfin molly (Poecilia latipinna) and a female Atlantic molly (Poecilia mexicana) about 100,000 generations ago [30, 31]. Although reproduction is clonal,

86 females require sperm from a male of one of the ancestral species (sailfin or Atlantic molly) to

87 initiate embryogenesis of unreduced ova [32]. Many distinct clonal lineages arose from the original diploid lineage through mutation or complete and/ or partial incorporation of paternal genetic material (i.e., through triploidy or acquisition of michrochromosomal-sub fragments of paternal chromosomes), which can be stable and transmitted to subsequent generations [32, 33].

91 Furthermore, new evidence shows high inter-clonal transcriptional variation which suggests

92 that different clonal lineages can adapt to different environments through long-term selection

93 on transcriptional fitness [34]. Together, this genetic diversity within a gynogenetic species

94 produces opportunities in which social interactions occur on multiple levels: within-clone

95 interactions, among-clone interactions, and interspecies interactions between Amazons and

96 their sexual hosts. While the interactions between Amazon mollies and their hosts has been the

97 focus of many investigations over the past forty years [e.g., 27, 335-38], little attention has

98 focused on the social interactions within and among the different clonal lineages. Nonetheless,

99 previous research suggests that clonal lineages vary in the social behaviors [39] and this

100 variation may be via functional differences in transcription landscapes [34].

101 In natural populations, the number of clonal lineages that co-occur can vary

102 dramatically from a single lineage to more than a dozen [40-42]. Therefore, the degree of

103 competition and the frequency with which females encounter conspecifics of different lineages

104 can vary greatly across time and space. One of the first studies to investigate social behaviors

105 among different clones reported that females could distinguish between lineages, associate

106 preferentially with fish of their own lineage, and were more aggressive toward unrelated clones

107 [39]. Other studies have reported that different features of the social environment can influence 
social behavior, especially aggression, within and among clonal lineages, including early

111 environment; that is, IGE are likely to be important regulators of phenotypic variation and

112 social dynamics in natural populations.

113 We leveraged clonal variation in Amazon mollies to test the hypothesis that IGE

114 propagate beyond individuals that experience them firsthand. This hypothesis predicts that

115 variation in behavior generated by IGE in a previous social environment will influence the

116 behavior of naïve individuals when an animal with this prior experience joins their group. To

117 distinguish this effect from first- and second-order IGE, we use the term 'cascading IGE'. Based

118 on extensive literature indicating that individual differences in behavior affects group-emergent

119 phenotypes [reviewed by 1, 2], we also predicted that cascading IGE would influence group-

120 emergent behavior in these fish. We tested these predictions by exposing genetically identical

121 Amazon mollies to social partners of different genotypes, and then moving these individuals to

122 new social groups in which they were the only member to have experienced IGE.

\section{2. Material and methods}

125 (a) Study Specimens

126 Three distinct clonal lineages were used in this study, but all lineages were descended

127 from individuals collected from the Río Purificacíon in Nuevo Padilla, Mexico $\left(24^{\circ} 4^{\prime} 42.85^{\prime \prime} \mathrm{N}\right.$,

$\left.1289^{\circ} 7^{\prime} 21.76^{\prime \prime} \mathrm{W}\right)$ originated from single-lineage stock populations kept in a greenhouse at the

129 Mission Road Research Facility of Florida State University. Both Clone 1 (VI/17 Schartl) and

130 Clone 2 (VI/17 AMM\#11) are diploid with microchromosomes, although the

131 microchromosomes are distinctly different between the two lineages [39, 46]. The focal clone

$132(\mathrm{VI} / 173 \mathrm{~N})$ is a triploid without any microchromosomes.

133 Two weeks prior to initiating the experiment, we marked all fish with elastomer tags

134 (two 3mm subcutaneous marks anterior and/or posterior of the dorsal fin) to allow us to 
135 identify focal individuals within each long-term social environment tank. To recover, fish were

136 placed in 113.6L aquaria treated with Stress Coat $+\circledR\left(\right.$ API () and sea salt (Instant Ocean $\left.{ }^{\circledR}\right)$, with

137 each aquarium containing an average of ten sister clone individuals. Females were all virgins

138 and, thus, were all receptive but not pregnant at the time of the trials. They were fed daily ad

139 libitum with commercial fish food (Tetramin ${ }^{\circledR}$ tropical flakes). Experiments occurred from

140 August to November 2019, and fish were exposed to natural light cycles during the course of

141 the experiment.

(b) Long-term social environments

Focal females were placed into 18.9L aquaria in one of three different long-term social environments: (1) 1 focal female +2 sister clones; (2) 1 focal female +2 females from a Clone 1; and (3) 1 focal female +2 females from Clone 2. That is, each aquarium contained 1 focal fish and 2 "social partner" fish. The partner fish genotypes, but not the genotype of focal fish,

148 differed among treatments. All aquaria contained sand and two small PVC pipe fittings $(2 \mathrm{~cm})$

149 for shelter, with one long side and two short sides covered with blue tarp to prevent visual 150 communication with neighboring tanks. Each social-environment treatment was replicated 12 151 times for a total of 36 experimental tanks. Experimental tanks were set up using a randomized 152 complete block design (one replicate of each treatment per block) over the course of two weeks 153 until all 12 blocks were complete. All females ranged between 27 and $38 \mathrm{~mm}$ in standard body 154 length with a maximum size difference among females within each social environment of $4 \mathrm{~mm}$.

155 To characterize differences in the social environment induced by the three different

156 social treatments, we measured social interactions in the experimental tanks at 9 different times 157 over the course of the experiment: $10 \mathrm{~min}$ after placing the focal fish in the social environment 158 (week 0), weekly for the first four weeks thereafter (weeks 1-4), and then biweekly until a total 159 of 12 weeks of exposure (weeks 6, 8, 10, and 12). Behavior measured at week 0 represents a 160 baseline because females had no prior exposure to social treatments at this time point. Social 161 behavior in the experimental tanks consisted mainly of aggressive interactions (bites, tail beats, 
162 and chasing); few affiliative or neutral behaviors (e.g., swimming in the same direction or

163 foraging simultaneously within 2 body lengths) were observed outside an aggressive context

164 (e.g., proceeding or following biting, chasing or tail beating). We counted the number of bites

165 and tail beats performed, and the total time (s) spent performing these behaviors and chasing

166 other females. Tail beats were rarer than bites, and the distribution was zero-inflated. We,

167 therefore, summed the total number bites and tail beats observed, and separately summed the

168 total time spent in aggressive interactions to produce two overall measures of aggression: total

169 number of aggressive acts and total time spent in aggression. Both measures were log-

170 transformed before analysis, after adding 1 to account for zero values. We excluded one datum

171 (block 7, clone 1 treatment, week 3) due to it being an extreme outlier: 174 aggressive acts (2.3x

172 higher than the maximum number of acts in any other tank), and 190s of total aggression (2.2x

173 larger than the maximum time spent being aggressive in any other tank). Individual

174 identification was not possible during the trial while fish were in motion and visible only from

175 one side. Therefore, we used the total number and duration of these behaviors across all fish in

176 the trial to characterize the social environment within the tank. These assays were recorded by a

177 live observer blind to the treatments for a duration of 10 minutes.

(c) Nä̈ve-group tests

Each focal female was introduced to a pair of novel ('naïve') social partners three times

181 over the course of the experiment (at 0, 4, and 12 weeks). A different pair of naïve social

182 partners was used at each of these trials, and those partner fish were not used with any other

183 focal female. We measured the average behavior of these naïve-groups before exposing focal

184 fish to genetically different long-term social environments (week 0) and after 4- and 12-weeks of

185 exposure (see figure 1). To do so, individual focal females were removed from their rearing tank

186 (at week 0) or their long-term social environment tank (at weeks 4 and 12) and placed in a

187 "naïve-group" test chamber with two unfamiliar females from the same clonal lineage as the

188 focal fish, size matched to the focal fish $( \pm 4 \mathrm{~mm})$, and in the same reproductive state. These 
novel fish were drawn from monoclonal, non-breeding rearing tanks similar to those from which focal and stimulus females originated and were, therefore, not exposed to the

191 experimental social environments experienced by the focal females. After we introduced the 192 focal fish into the naïve-group test chamber, we video recorded all three fish for 10 minutes,

193 after which the focal female was removed and placed back into her experimental social 194 environment (figure 1).

195 The naïve-group test chamber was an open field, circular tank (55.9 cm diameter), with 196 half the bottom and corresponding sides painted white and the other half grey. This test 197 chamber was placed inside a frame covered with blue tarp to minimize external disturbance. All 198 water in the chamber was replaced with clean freshwater prior to every test. In the center of the 199 frame, a camera (JVC Everio 1920x1080 HD video camcorder) was suspended $1.1 \mathrm{~m}$ above the 200 tank. All videos were edited to remove the first and last 2 minutes of recording (VideoPad 201 Video Editor by $\mathrm{NCH}$ sofware $\mathrm{C}, \mathrm{v}$. 8.40) to allow for acclimation to the experimental tank and 202 to remove any influence of camera or experimenter movement at the beginning and end of trial. 203 All cropped videos were 6 minutes long and were analyzed by a blind observer using 204 EthoVision XT (Noldus, v14). Within the EthoVision program, we distinguished the three 205 individuals throughout the analyses and acquired movement and position data (Cartesian 206 coordinates) for all three individuals. Although fish could be individually tracked, the focal 207 individual could not be distinguished from the novel partner fish on the videos; therefore, we 208 did not calculate separate metrics for focal and novel partner fish. We extracted the following 209 measures from EthoVision: distance traveled $(\mathrm{cm})$, velocity $\left(\mathrm{cm} / \mathrm{s}^{2}\right)$, frequency entering white 210 zone, duration in white zone (s), latency to enter white zone (s), frequency entering grey zone, 211 duration in grey zone (s), time spent immobile (s; freezing behavior), and distance between 212 individuals (cm; shoaling distance).

213 We interpret these behaviors to reflect stress-related behavior and tendency to be 214 exploratory. More stressed individuals are less active, travel shorter distances at lower velocity, 215 spend more time frozen and in the grey zone (negative phototaxis), and are closer together; less 
216 stressed individuals tend to be more exploratory and cover more distance, move at higher

217 velocity, enter zones more frequently, spend more time in the white zone and less time frozen,

218 and haver more distance between individuals $[47,48]$. We also gathered baseline data on these

219 behaviors by following the same procedure at the start of the experiment, before the focal fish

220 had experienced the experimental social treatments (time 0; figure 1: Pre-exposure).

(d) Ethics

All fish tanks included substrate and enrichment and were maintained with weekly

224 water changes throughout the duration of this study. Fish never suffered from food deprivation 225 or injuries during this study. This research was approved by the Institutional Animal Care and 226 Use Committee of Florida State University (1704 and 201900038).

(e) Analyses

There were no significant differences in size (SL) among focal females in different social treatment groups, nor treatment-associated differences in size among the social partners fish

231 used in the long-term and naïve-group trials (electronic supplemental material, table S1).

232 Nevertheless, we included SL of focal and partner fish as covariates in subsequent analyses

233 because there was a non-significant trend for Clone 1 and Clone 2 social partner fish to differ in

234 SL (electronic supplemental material, table S2)

236 (e.1) Long-term social environment groups.

237 We assessed the correlation structure of the two measurements of aggression to 238 determine if they could be adequately represented by principal components (PC), and then 239 used the first PC from this analysis as our measure of aggression (see Results). To determine if 240 aggression was influenced by social treatment group, we used this PC score as the dependent 241 variable in general linear mixed models that accounted for the repeated measures on each 242 group. In addition to the social treatment group, initial models included fixed effects of 
243 exposure time (weeks), treatment-by-time interaction, the baseline (week 0) measure of

244 aggression PC1 in each group, focal female standard length (log-transformed), and the average

245 standard length of the social-partner females (log-transformed). A random within-subjects

246 effect with group ID as the subject was used to account for repeated measures on groups; initial

247 models also included a random effect due to experimental block. The fixed effects of baseline

248 aggression and size of social partners never approached significance in initial models (electronic

249 supplemental material, table S3A), and the random block effect was consistently near zero and

250 never significant. These terms were, therefore, not included in the final models. This and other

251 analyses of general linear mixed models were conducted using SAS Proc Glimmix in SAS v. 9.4

252 (SAS Institute Inc. 2013. SAS/STAT 9.4. SAS Institute Inc., Cary, NC.). Because repeated

253 observations were not equally spaced in time, we used a covariance structure that allows for

254 unequally-spaced observations (a 1-dimensional spatial structure, implemented with the

$255 s p$ (pow) option). Within-subject variance estimates were allowed to vary by treatment group, by

256 using the group option. Post hoc comparisons of treatment group means were conducted using

257 the simulation method of [49], as implemented by using the adjust=simulate option.

(e.2) Naïve-group tests.

To determine the extent to which the presence of the focal individual influenced

261 behavior in the naïve-groups, and thus to measure cascading IGE, we calculated two kinds of

262 metrics: those that described average behavior of the 3 members of the group, and those that

263 described individual behavior of fish within the group. For both analyses, we included seven of

264 the movement variables (distance traveled $(\mathrm{cm})$, velocity $\left(\mathrm{cm} / \mathrm{s}^{2}\right)$, frequency entering white

265 zone, duration in white zone (s), latency to enter white zone (s), time spent immobile (s; freezing

266 behavior), and distance between individuals (cm; shoaling distance)); the frequency entering

267 and duration in the gray zone was redundant with information for entering and duration in the

268 white zone, so we used only the data for the white zone in the analyses. 
to determine if they could be adequately represented by principal components (PC). The six

271 behaviors that described movement or physical position in the enclosure were all moderately to

272 highly correlated with one another $(0.4<|\mathrm{r}|<1.0)$, but they were not correlated with the

273 average shoaling distance between fish (all $|\mathrm{r}|<0.2)$ (electronic supplementary material, figure

274 S1A), indicating that a PCA should include the 6 movement/position variables, but that

275 shoaling distance should be analyzed separately. We used the first PC from this analysis as our

276 measure of the movement and position of fish (see Results), and we used the log-transformed

277 average shoaling distance as a measure of a group cohesion, since it arises from the relative

278 positions of all three members of the group.

To determine if these two measures of naïve-group behavior were affected by the social

280 environment experienced by a single member of the group, we used them as dependent

281 variables in general linear mixed models that accounted for repeated measures on a group,

282 implemented in SAS Proc Glimmix. Initial models included fixed effects of the long-term social

283 environment of the focal fish, time in the long-term social environment, size of the focal female,

284 the mean size of the novel partner females, mean size of the long-term social partners, and

285 baseline behavior (i.e., before exposure of the focal female to long-term social environments). To

286 assess whether the effect of the long-term social environment was mediated by aggression

287 experienced by the focal female, initial models also included a measure of aggression averaged

288 over the 4 weeks prior to the naïve-group test (PC1 of aggressive behaviors averaged over those

2894 weeks). A within-subjects random effect with focal female ID as the subject was used to

290 account for the three different naïve-group trials in which each focal female was used; initial

291 models also included a random effect due to experimental block. Neither the size-related fixed

292 effects nor the summary measure of aggression ever approached significance in the initial

293 models (electronic supplemental material, table S3B and C), so only treatment and exposure

294 time (and their interaction) were retained in the final models. The block random effect was

295 always near zero and never approached significance, so it was dropped from the final models. 
296 We used the compound symmetry covariance structure because it fit the data better than

297 alternative structures (by AICC and BIC metrics). Post hoc comparisons of group means were 298 conducted as described above.

299 Behavior of individuals in naïve-groups. The main purpose of this analysis was to determine

300 if differences in the average behavior among groups was attributable to all members of a group

301 behaving similarly or to specific individuals within the group. For example, if the behavior of

302 the three females within a group was very similar, then average differences among groups

303 reflect the behavior of all group members. Alternately, if individuals within groups behaved

304 differently from one another, then between-group differences could have been driven by the

305 divergent behavior of a single group member. The former, but not the latter would support

306 cascading IGE because it would indicate that non-focal behavior was influenced by the prior

307 social experience of the focal fish. Our primary measure of similarity of the behavior of

308 individuals within naïve-groups was the intraclass correlation coefficient (ICC). ICC values near

3091 indicate that individuals within a group behaved very similarly to one another, whereas lower

310 values of ICC indicate substantial differences in behavior among members of the group.

311 We first investigated the correlation structure of the same 8 behaviors described above

312 but measured on individuals rather than the mean of the 3 fish in a group. Similar to the group-

313 average data, the position/movement variables were moderately to highly correlated with each

314 other, but not with shoaling distance (electronic supplementary material, figure S1B). We,

315 therefore, summarized the movement/position behavior of individual fish using the first PC of

316 the 6 movement/position metrics (table 1). As in the group-averaged data, behaviors associated

317 with exploration loaded positively on PC1 (distance, velocity, and duration and frequency in

318 the white zone), while behaviors associated with reluctance to explore loaded negatively on

319 PC1 (freezing, latency to enter white zone, table 1). Again, we considered positive values of the

320 first PC to indicate a tendency to be exploratory and negative values a tendency to be stressed.

321 We used the log-transformed individual shoaling distance (the mean distance of a single

322 individual from her group mates during a trial) as a measure of individual tendency to shoal. 
323 We then calculated the ICC of the individual exploratory behavior scores and the individual

324 shoaling distances as the ratio of the between-group variance to the total variance. These

325 variance components were calculated using SAS Proc Mixed with default settings and a single

326 random effect corresponding to the group ID.

\section{Results}

(a) High correlations found within the aggression behaviors and among the movement/position variables

We found that the two measures of aggression (number of acts and time spent) were

331 highly correlated $\left(\mathrm{R}^{2}=0.803, \mathrm{p}<0.0001\right)$, with the first PC explaining $96.9 \%$ of the total variation

332 (electronic supplementary material, figure S2A). For the average behaviors of the naïve-groups,

333 the first PC summarizing the 6 movement/position variables explained $72.4 \%$ of the total

334 variation, and it was the only PC with an eigenvalue $>1$ (table 1, electronic supplementary

335 material, figure S2B). In this PCA, behaviors associated with exploration loaded positively on

336 PC1 (distance, velocity, and duration in the white zone, and frequency entering white zones),

337 while behaviors associated with stress loaded negatively on PC1 (freezing, latency to enter

338 white zone, table 1). We therefore considered positive values of PC1 to indicate a tendency to

339 explore, and negative values to indicate lack of exploration or stress-like behaviors.

(b) Long-term social environments differ in social behavior

Long-term social groups in which the focal fish was housed with two females of its own

343 clonal lineage exhibited more aggression than groups where the social partners were Clone 1 or

344 Clone 2 fish (figure 2A, table 2A, effect estimates provided in electronic supplementary

345 material, table S4). On average, fish in the Monoclonal environment performed 60\% more

346 aggressive acts than fish in the Clone 1 environment (14.45 \pm 1.49 vs. $9.04 \pm 1.27$ acts per 10-

347 minute observation bout, respectively; fish in the Clone 2 environment performed $11.04 \pm 1.18$

348 aggressive acts per bout, on average). Post hoc tests indicated that the Monoclonal social

349 environment elicited significantly more aggressive behavior than the Clone 1 environment 
$350(\mathrm{p}<0.001)$, but no other contrasts were significant after adjustment for multiple tests

351 (Monoclonal vs Clone 2: $\mathrm{p}=0.087$; Clonal 1 vs Clone 2: $\mathrm{p}=0.114$; table 2A; electronic

352 supplementary material, figure S3).

(c) Genetic differences in prior social experience for one group member affected behavior of all members of

355 the naïve-groups.

The long-term social environment experienced by the single focal fish in a naïve-group

affected the exploratory behavior of the entire group (table $2 \mathrm{~B}$, figure $2 \mathrm{~B}$, and electronic

supplementary material, figure S4, effect estimates for fixed effects provided in electronic

supplementary material, table S5). Indeed, the social environment explained $43.1 \%$ of the total

variation in the exploratory/stress PC1 scores [50]. This result is particularly striking because all

361 focal and stimulus fish were members of a single clonal linage and, therefore, genetically

362 identical [39]. Specifically, groups in which the focal individual experienced the Monoclonal

363 long-term social environment exhibited more stress-related behavior (negative values on

364 exploratory PC1) than groups in which the focal individual experienced Clone 1 or Clone 2

365 social environments (post-hoc tests: Monoclonal vs Clone 1, p=0.021; Monoclonal vs Clone 2,

$366 \mathrm{p}=0.004)$. Naïve-groups in which the focal fish had experienced social environments containing

367 Clone 1 and Clone 2 did not differ from each other after correction for multiple tests $(p=0.259)$.

The mean shoaling distance in the naïve-groups was unaffected by the social

369 environment experienced by the focal fish, duration of exposure, or their interaction (table 2C,

370 effect estimates for fixed effects provided in electronic supplementary material, table S5, figure

371 S5).

372

(d) Individuals within naïve-groups behave very similarly.

Focal and stimulus fish within the naïve-groups were unfamiliar with one another and

375 had different social experiences prior to the trials. Focal fish were drawn from the long-term

376 social environments, whereas stimulus fish were all genetically identical, all of similar age and 
size, and all had similar prior social experience that differed substantially from that of the focal fish. Moreover, there was substantial variation in behavior across different trials, as indicated by the significant effects of long-term social environment described above. Nevertheless, the three individuals in a given trial behaved in a remarkably similar manner (ICC for individual exploratory behavior: 0.913; ICC for individual shoaling distance: 0.953). Figure 3 shows representative tracking data for 3 different trios from the naïve-group tests (see electronic supplementary material, figure S6 for 12 additional representations). The striking visual similarity of tracking patterns within a given trial is reflected in very high ICC estimates across each treatment for both individual exploratory behavior (Monoclonal: 0.917; Clone 1: 0.924;

Clone 2: 0.897) and for individual shoaling distance (Monoclonal: 0.941; Clone 1: 0.970; Clone 2: material, table S7. That is, less than $10 \%$ of the total variation in behavior occurred among the three females within a given trial, despite the substantial differences in behavior among trials that is evident in figure 3 and electronic supplementary material, figure S6. These high ICC

391 values indicate that all three individuals within a given trial exhibited highly similar behavior,

392 despite their different prior experience.

\section{Discussion}

Elucidating the heritable causes of individual and group-level behavior is necessary to understand the evolution of social traits. Here, we demonstrate that phenotypic effects of genetically different social environments (IGE) carry over to a novel social environment to

398 influence the behavior of individuals that did not experience IGE. This cascading effect is 399 distinct from 'second-order' IGE [20,21], in which the presence of genetically different 400 individuals influences interactions between other group members. Our results, therefore, 401 expand the scope of IGE by demonstrating that they can influence phenotypes even when there 402 is no genetically-based variation present within groups. Given the prevalence of dispersal and 403 fission-fusion social structure, there is substantial opportunity for cascading IGE in nature. 

analysis, or transplant experiments, could reveal effects that related individuals have on new social partners, but this would require tracking individuals that disperse into new social groups. Such investigations can be facilitated by using species with clonal reproduction (e.g., many microbes and plants, and some vertebrates and invertebrates). In these systems, high relatedness within clones will make cascading IGE easier to detect. Sexual species in which

411 relatedness is known and social interactions can be recorded after dispersal from natal groups 412 could provide additional opportunities to measure cascading IGE.

414 focal fish experienced in the long-term social environments. Somewhat surprisingly, it was the

415 social environment containing fish of the same clone as the focal animals that exhibited the most

416 aggression (and the naïve-groups containing these focal fish exhibited the most stress

417 behaviors). Previous studies found that Amazon mollies exhibited less aggression towards 418 sister clones when compared to non-sister clones [39, 51]. However, a different focal clonal 419 lineage was used in those studies, suggesting that responses to sister and non-sister clones (and, 420 therefore, first-order and cascading IGE) vary across genotypes. Consequently, we predict that 421 higher levels of aggression within the social environment result in more stressed individuals, 422 regardless of whether that social environment is monoclonal or composed of non-sister clones;

423 thus, fish should exhibit less exploratory behaviors when in new social group. Furthermore, this

424 kind of interaction between the direct effect of an individual's genotype and IGE can produce

425 frequency-dependent and other forms of balancing selection that can maintain, or rapidly erode 426 genetic variation $[52,53]$. The possibility that similar effects could arise from the interaction of 427 direct genetic variance and cascading IGE warrants future empirical and theoretical 428 investigation. 
431 social environment when they moved into the naïve-groups, but focal fish from the Monoclonal

432 treatment did not. This would predict a significant difference between the Monoclonal

433 treatment and both Clone 1 and Clone 2 (which we do find), but not between Clone 1 and Clone

4342 (for which we found only a non-significant trend). Nevertheless, our data support the

435 conclusion that genetically identical fish (the naïve partners) behave differently depending on

436 genetic variation in the prior social environment experienced by another member of the group

437 (the focal female). Whether cascading IGE depend on the degree of genetic similarity between

438 past and current social partners should be a focus of future research.

439 We detected no effects of exposure time within the long-term social environments on

440 aggression in those environments or on cascading IGE in the naïve-groups. Time-course effects

441 on first-order IGE have been found in mosquitofish [53,54], and increased exposure time led to

442 higher aggression in previous studies of Amazon mollies [44, 45]. However, the time course

443 effects of IGE reported in mosquitofish occurred during maturation, whereas the fish in our

444 experiment were fully mature at the start of the study. The two studies that reported exposure-

445 time effects on aggression in Amazon mollies maintained the animals at considerably higher

446 density than that used in our experiment (44: 1.9 L / fish; 45: 4 L / fish; the present study: $6.3 \mathrm{~L}$

447 / fish), suggesting that exposure-time effects could be density-dependent.

448 The relatively low density in our long-term social environments might also account for

449 lack of treatment or cascading effects on shoaling distance, despite strong effects on exploratory

450 behavior. Anderson et al. [21] found that second-order IGE influenced social cohesion in D.

451 melanogaster, and the extensive literature on leadership in social organisms indicates that

452 differences among individual group members can substantially influence group-emergent

453 behaviors such as shoaling [55, reviewed in 1]. We, therefore, predict that cascading IGE could

454 be an important source of individual variation that generates group-emergent phenotypes [26,

455 56]. In our experiment, groups consisted of only 3 individuals, which might limit the tendency

456 of these fish to shoal. Experiments that use larger groups and enclosures that allow more 
flexibility in fission-fusion dynamics could determine the extent to which cascading IGE

influence group-emergent phenotypes.

In summary, IGE propagate beyond individuals that directly experience them in

460 Amazon mollies and possibly in many group-living species. These cascading IGE are a

461 potentially important cause of individual differences that can lead to the emergence of leaders

462 and followers, shoaling, swarming, and other group-emergent phenotypes. Theoretical and

463 empirical expansion of the robust quantitative genetic framework developed for IGE to include

464 cascading or other types of carry-over effects will facilitate understanding of social behavior

465 and its evolution.

\section{References}

1. del Mar Delgado M, Miranda M, Alvarez SJ, Gurarie E, Fagan WF, Penteriani V, di Virgilio A, Morales JM. 2018 The importance of individual variation in the dynamics of animal collective movements. Philos. Trans. R. Soc. Lond. B. Bio.l Sci. 373, 2017008.

2. Jolles JW, King AJ, Killen SS. 2020 The Role of Individual Heterogeneity in Collective Animal Behaviour. Trends Ecol. Evol. 35, 278-291.

3. Moore AJ, Brodie III ED, Wolf JB. 1997 Interacting phenotypes and the evolutionary process: I. Direct and indirect genetic effects of social interactions. Evolution 51, 1352-1362.

4. Wolf JB, Brodie III ED, Cheverund JM, Moore AJ, Wade MJ. 1998 Evolutionary consequences of indirect genetic effects. Trends Ecol. Evol. 13, 64-69.

5. Bijma P, Wade MJ. 2008 The joint effects of kin, multilevel selection and indirect genetic effects on response to genetic selection. J. Evol. Biol. 21, 1175-1188.

6. Mutic JJ, Wolf JB. 2007 Indirect genetic effects from ecological interactions in Arabidopsis thaliana. Mol. Ecol. 16, 2371-2381.

7. Hunt J, Simmons LW. 2002 The genetics of maternal care: Direct and indirect genetic effects on phenotype in the dung beetle Onthophagus taurus. Proc. Natl. Acad. Sci. USA 99, 68286832.

8. Wilson AJ, Gelin U, Perron M-C, Réale D. 2009 Indirect genetic effects and the evolution of aggression in a vertebrate system. Proc. R. Soc. B. 276, 533-541.

9. Bleaker BH, Brodie III ED. 2008 Indirect genetic effects influence antipredator behavior in guppies: Estimates of the coefficient of interaction Psi and the inheritance of reciprocity. Evolution 63, 1796-1806.

10. McFarlane SE, Gorrell JC, Coltman DW, Humphries MM, Boutin S, McAdam AG. 2015 The nature of nurture in a wild mammal's fitness. Proc. R. Soc. B. 282, 20142422.

11. Santostefano F, Wilson AJ, Niemela PT, Dingemanse NJ. 2017 Indirect genetic effects: A key component of the genetic architecture of behaviour. Sci. Rep. 7, 10235.

12. Alemu SW, Bijma P, Møller SH, Janss L, Berg P. 2014 Indirect genetic effects contribute substantially to heritable variation in aggression-related traits in group-housed mink (Neovison vison). Genet. Sel. Evol. 46, 30.

13. Camerlink I, Turner SP, Bijma P, Bolhuis JE. 2013 Indirect genetic effects and housing conditions in relation to aggressive behaviour in pigs. PLoS ONE 8, e65136. 
14. Baud A, Casale FP, Nicod J, Stegle O. 2020 Dissecting the mechanisms underlying indirect genetic effects on biomedical phenotypes: a study on 170 behavioural, physiological and morphological phenotypes measured in adult laboratory mice. bioRxiv 302349. https: / / doi.org/10.1101/302349.

15. Fisher DN, Wilson AJ, Boutin S, Dantzer B, Lane JE, Coltman DW, Gorrell JC, McAdam AG. 2019 Social effects of territorial neighbours on the timing of spring breeding in North American red squirrels. J. Evol. Biol. 32, 559-571.

16. Peeters K, Eppink TT, Ellen ED, Visscher J, Bijma P. 2012 Indirect genetic effects for survival in domestic chickens (Gallus gallus) are magnified in crossbred genotypes and show a parent-of-origin effect. Genetics 192, 705-713.

17. Wolf JB, Moore AJ. 2010 Interacting phenotypes and indirect genetic effects: a genetic perspective on the evolution of social behavior. In Evolutionary Behavioral Ecology, pp. 225245. New York: Oxford University Press.

18. Bailey NW, Marie-Orleach L, Moore AJ, Simmons L. 2018 Indirect genetic effects in behavioral ecology: does behavior play a special role in evolution? Behav. Ecol. 29, 1-11.

19. Bailey NW, Kolliker M. 2019 Social runaway: Fisherian elaboration (or reduction) of socially selected traits via indirect genetic effects. Evolution 73, 1549-1563.

20. Saltz JB. 2013 Genetic composition of social groups influences male aggressive behaviour and fitness in natural genotypes of Drosophila melanogaster. Proc. R. Soc. B. 280, 20131926.

21. Anderson BB, Scott A, Dukas R. 2017 Indirect genetic effects on the sociability of several group members. An. Behav. 123, 101-106.

22. Jolles JW, Boogert NJ, Sridhar VH, Couzin ID, Manica A. 2017 Consistent individual differences drive collective behavior and group functioning of schooling fish. Curr. Biol. 27, 2862-2868.

23. Ancillotto L, Allegrini C, Serangeli MT, Jones G, Russo D. 2014 Sociality across species: spatial proximity of newborn bats promotes heterospecific social bonding. Behav. Ecol. 26, 293-299.

24. Ruploh T, Henning M, Bischof HJ, von Engelhardt N. 2015 Effects of social conditions during adolescence on courtship and aggressive behavior are not abolished by adult social experience. Dev. Psychobiol. 57, 73-82.

25. Cox JA, Cusick JA, DuVal EH. 2019 Manipulated sex ratios alter group structure and cooperation in the brown-headed nuthatch. Behav. Ecol. 30, 883-893.

26. Jolles JW, Weimar N, Landgraf T, Romanczuk P, Krause J, Bierbach D. 2020. Group-level patterns emerge from individual speed as revealed by an extremely social robotic fish. Bio. Let. 20200436.

27. Jolles JW, Fleetwood-Wilson A, Nakayama S, Stumpe M, Johnstone RA, Manica A. 2014 The role of previous social experience on risk-taking and leadership in three-spine sticklebacks. Behav. Ecol. 25, 1395-1401.

28. Jolles JW, Taylor BA, Manica A. 2016 Recent social conditions affect boldness repeatability in individual sticklebacks. An. Behav. 112, 139-145.

29. Hubbs CL, Hubbs LC. 1932 Apparent parthenogenesis in nature in a form of fish of hybrid origin. Science 76, 628e630.

30. Avise JC, Trexler JC, Travis J, Nelson WS. 1991 Poecilia mexicana is the recent female parent of the unisexual fish $P$. formosa. Evolution 45, 1530-1533.

31. Stöck M, Lampert KP, Möller D, Schlupp I, Schartl M. 2010 Monophyletic origin of clonal lineages in an asexual fish (Poecilia formosa). Mol. Ecol. 19, 5204-5215.

32. Schartl M, Nanda I, Schlupp I, Wilde B, Eppens JT, Schmid M, Parzefall J. 1995 Incorporation of subgenomic amounts of DNA as compensation for mutational load in a gynogenetic fish. Nature 373, 68-71.

33. Schlupp I, Riesch R. 2011 Evolution of unisexual reproduction. In Ecology and Evolution of Poeciliid Fishes (eds J Evans, A Pilastro, I Schlupp), pp. 50-58. Chicago, IL: University of Chicago Press. 
34. Lu Y, Bierbach D, Ormanns J, Warren WC, Walter RB, Schartl M. 2021 Fixation of allelic gene expression landscapes and expression bias pattern shape the transcriptome of the clonal Amazon molly. Genome Res. 31, 1-8.

35. Balsano JS, Randle EJ, Rasch EM, Monaco PJ. 1985 Reproductive behavior and the maintenance of all-female Poecilia. Environ. Biol. Fishes 12, 251-263.

36. Schlupp I, Marler C, Ryan MJ. 1994 Benefit to male sailfin mollies of mating with heterospecific females. Science 263, 373-374.

37. Heubel KU, Hornhardt K, Ollmann T, Parzefall J, Ryan MJ, Schlupp I. 2008 Geographic variation in female mate-copying in the species complex of a unisexual fish, Poecilia formosa. Behaviour 145, 1041-1064.

38. Makowicz AM, Murray L, Schlupp I. 2020. Size, species, and audience type influences heterospecific female-female competition. An. Behav. 159, 47-58.

39. Makowicz AM, Tiedemann R, Steele RN, Schlupp I. 2016 Kin recognition in a clonal fish, Poecilia formosa. PLoS ONE 11, e0158442.

40. Kallamn KD. 1962 Population genetics of the gynogenetic teleost, Mollienesia formosa (Girard). Evolution 16, 497-504.

41. Darnell RM, Lamb E, Abramoff P. 1965 Matroclinous inheritance and clonal structure of a Mexican population of the gynogenetic fish, Poecilia formosa. Evolution 21, 168-173.

42. Lamatsch DK, Nanda I, Schlupp I, Epplen JT, Schmid M, Schartl M. 2004 Distribution and stability of supernumerary microchromosomes in natural populations of the Amazon molly, Poecilia formosa. Cytogenet. Genome Res. 106, 189-194.

43. Laskowski KL, Wolf M, Bierbach D. 2016 The making of winners (and losers): How early dominance interactions determine adult social structure in a clonal fish. Proc. R. Soc. B. 283, 20160183.

44. Makowicz AM, Schlupp I. 2015 Effects of female-female aggression in a sexual/unisexual species complex. Ethology 121, 904-914.

45. Doran C, Bierbach D, Laskowski K. 2019 Familiarity increases aggressiveness among clonal fish. An. Behav. 148, 153-159.

46. Warren WC, García-Pérez R, Xu S, Lampert KP, Chalopin D, Stöck M, Loewe L, Lu Y, Kuderna L, Minx P, et al. 2018 Clonal polymorphism and high heterozygosity in the celibate genome of the Amazon molly. Nat. Ecol. Evol. 2, 669-679.

47. Cachat JM, Canavello PR, Elkhayat SI, Bartels BK, Hart PC, Elegante MF, Beeson EC, Laffoon AL, Haymore WAM, Tien DH, et al. 2011 Video-aided analysis of zebrafish locomotion and anxiety-related behavioral responses. In Zebrafish neurobehavioral protocols (eds AV Kalueff, JM Cachat), pp. 1-14. New York, USA: Humana Press.

48. Baker MR, Goodman AC, Santo JB, Wong RY. 2018 Repeatability and reliability of exploratory behavior in proactive and reactive zebrafish, Danio rerio. Sci. Rep. 8, 12114.

49. Edwards D, Berry JT. 1987 The efficiency of simulation-based multiple comparisons. Biometrics 43, 913-928.

50. Jaeger BC, Edwards LJ, Das K, Sen PK. 2016 An R2 statistic for fixed effects in the generalized linear mixed model. J Appl. Stat. 44, 1086-1105.

51. Makowicz AM, Moore T, Schlupp I. 2018 Clonal fish are more aggressive to distant relatives especially when hungry. Behaviour 155, 351-367.

52. Wolf JB. 2000 Indirect Genetic Effects and Gene Interactions. In Epistasis and the Evolutionary Process (eds JB Wolf, ED Brodie, MJ Wade), pp. 158-176. Oxford: Oxford University Press.

53. Culumber ZW, Kraft B, Lemakos V, Hoffner E, Travis J, Hughes KA. 2018 GxG epistasis in growth and condition and the maintenance of genetic polymorphism in Gambusia holbrooki. Evolution 72, 1146-1154.

54. Kraft B, Lemakos VA, Travis J, Hughes KA. 2018 Pervasive indirect genetic effects on behavioral development in polymorphic eastern mosquitofish. Behav. Ecol. 29, 289-300.

55. Bierbach D, Langraf T, Romanczuk P, Lukas J, Nyguyen H, Wolf M, Krause J. 2018. Using a robotic fish to investigate individual differences in social responsiveness in the guppy. $R$. Soc. open sci. 5, 181026. 
603 56. Herbert-Read JE, Perna A, Mann RP, Schaef TM, Slumpter DJT, Ward AJW. 2011. Inferring

604 the rules of interaction of shoaling fish. Proc. Natl. Acad. Sci. USA 108, 18726-18731.

605

\section{Acknowledgements}

607 We would like to thank Mitch Daniel, Kevin Dixon, and Alexa Guerrera for their input on a

608 previous version of this manuscript and Ryan Kelly, Hannah Lange, and Jacob Gottlieb for

609 assistance with the behavioral setup and fish maintenance. This research was approved by the

610 Florida State University's Institutional Animal Care and Use Committee (\#1704 and 201900038).

611 AMM was supported by Provost Postdoctoral Fellowship Program, DB was funded by the

612 German Research Foundation (DFG) through Germany's Excellence Strategy (EXC 2002/1

613 'Science of Intelligence', project number 390523135), and KH was funded by NSF IOS 1354775

614 and NSF DEB 1740466. 
615 Table 1. PC loadings for PC1 on group-averaged and individual-level exploratory/stress

616 behaviors.

617

\begin{tabular}{lllr}
\hline Level & Model & Measurement & PC1 loading \\
\hline Group-averaged & PC1 exploratory/stress & Total distance traveled & 0.4571 \\
& & Velocity & 0.4565 \\
& & Frequency entering white zone & 0.4516 \\
& & Duration in white zone & 0.3237 \\
& & Latency to enter white zone & -0.3053 \\
& & Time spent frozen in place & -0.4251 \\
& & \\
Individual-level & PC1 exploratory/stress & Total distance traveled & 0.4716 \\
& & Velocity & 0.4711 \\
& & Frequency entering white zone & 0.4484 \\
& & Duration in white zone & 0.3080 \\
& & Latency to enter white zone & -0.2605 \\
& & Time spent frozen in place & -0.4380 \\
\hline
\end{tabular}

618 
619 Table 2: Test statistic and p-value for statistical model of aggression PC1, exploratory/stress

620 behaviors (PC1), and shoaling behaviors as dependent variables.

\begin{tabular}{|c|c|c|c|}
\hline Model & Effect & Statistic & P-value \\
\hline \multicolumn{4}{|c|}{ A. Aggressive behavior in long-term social treatments (PC1) } \\
\hline & Focal female standard length & $\mathrm{F}_{1,208.1}==3.26$ & 0.072 \\
\hline & Social environment & $F_{2,250.3}=7.70$ & $<0.001$ \\
\hline & Exposure time & $\mathrm{F}_{7,221.2}=1.30$ & 0.253 \\
\hline & Social environment ${ }^{*}$ Time & $\mathrm{F}_{14,232.6}=0.69$ & 0.788 \\
\hline \multicolumn{4}{|c|}{ B. Exploratory/stress behavior in nä̈ve-group trials (PC1) } \\
\hline & Social environment & $F_{2,19.91}=7.55$ & 0.004 \\
\hline & Exposure time & $\mathrm{F}_{1,23.29}=0.55$ & 0.466 \\
\hline & Social environment ${ }^{\star}$ Time & $\mathrm{F}_{2,16.03}=0.78$ & 0.476 \\
\hline \multicolumn{4}{|c|}{ C. Shoaling distance in naïve-group trials } \\
\hline & Social environment & $F_{2,20.04}=0.60$ & 0.560 \\
\hline & Exposure time & $\mathrm{F}_{1,26.93}=0.07$ & 0.793 \\
\hline & Social environment*Time & $\mathrm{F}_{2,20.1}=3.20$ & 0.062 \\
\hline
\end{tabular}

621 
622 Figure 1: Schematic of experimental design illustrating the focal females $\left(\mathrm{FF}_{\mathrm{CL}}\right)$ tested for pre-

623 exposure exploratory behaviors with two novel sister clones $\left(\mathrm{F}_{\mathrm{CL}}\right)$ at week 0 . Focal females were

624 then transferred into one of the three different social environments: Monoclonal $\left(\mathrm{FF}_{\mathrm{CL}}+2 \mathrm{~F}_{\mathrm{CL}}\right)$;

625 Clone $1\left(\mathrm{FF}_{\mathrm{CL}}+2 \mathrm{CL}_{1}\right)$; or Clone $2\left(\mathrm{FF}_{\mathrm{CL}}+2 \mathrm{CL}_{2}\right)$. After 4 and 12 weeks of exposure to these

626 social environments, the exploratory behaviors of the focal females were tested again with novel

$627 \quad \mathrm{~F}_{\mathrm{CL}}$ individuals. Note that the $\mathrm{F}_{\mathrm{CL}}$ partners of the $\mathrm{FF}_{\mathrm{CL}}$ were different individuals at each time

628 period. That is, each individual $\mathrm{F}_{\mathrm{CL}}$ was included in only one trial.

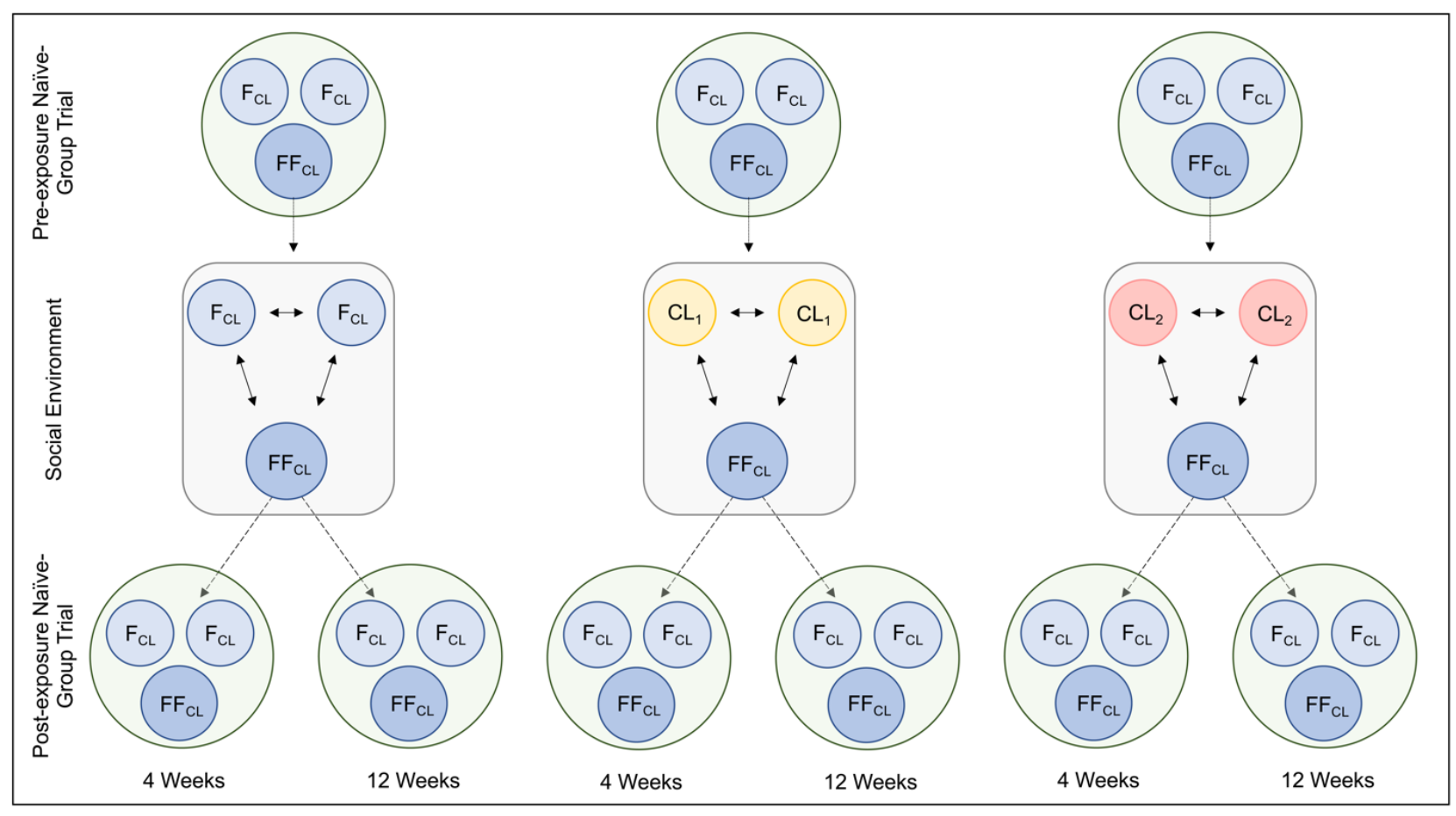


629 Figure 2: Least square means + /- standard error from PC1 for (A.) aggression focal females

630 were exposed to in each social environment (Monoclonal (blue); Clone 1 (yellow); Clone 2

631 (pink)). Positive values indicate more aggression. (B.) Exploratory/stress behaviors in the naïve-

632 group trials. Group-averaged exploratory behaviors with positive values indicating more

633 exploratory behaviors and negative values indicate less exploratory and more stress behaviors.

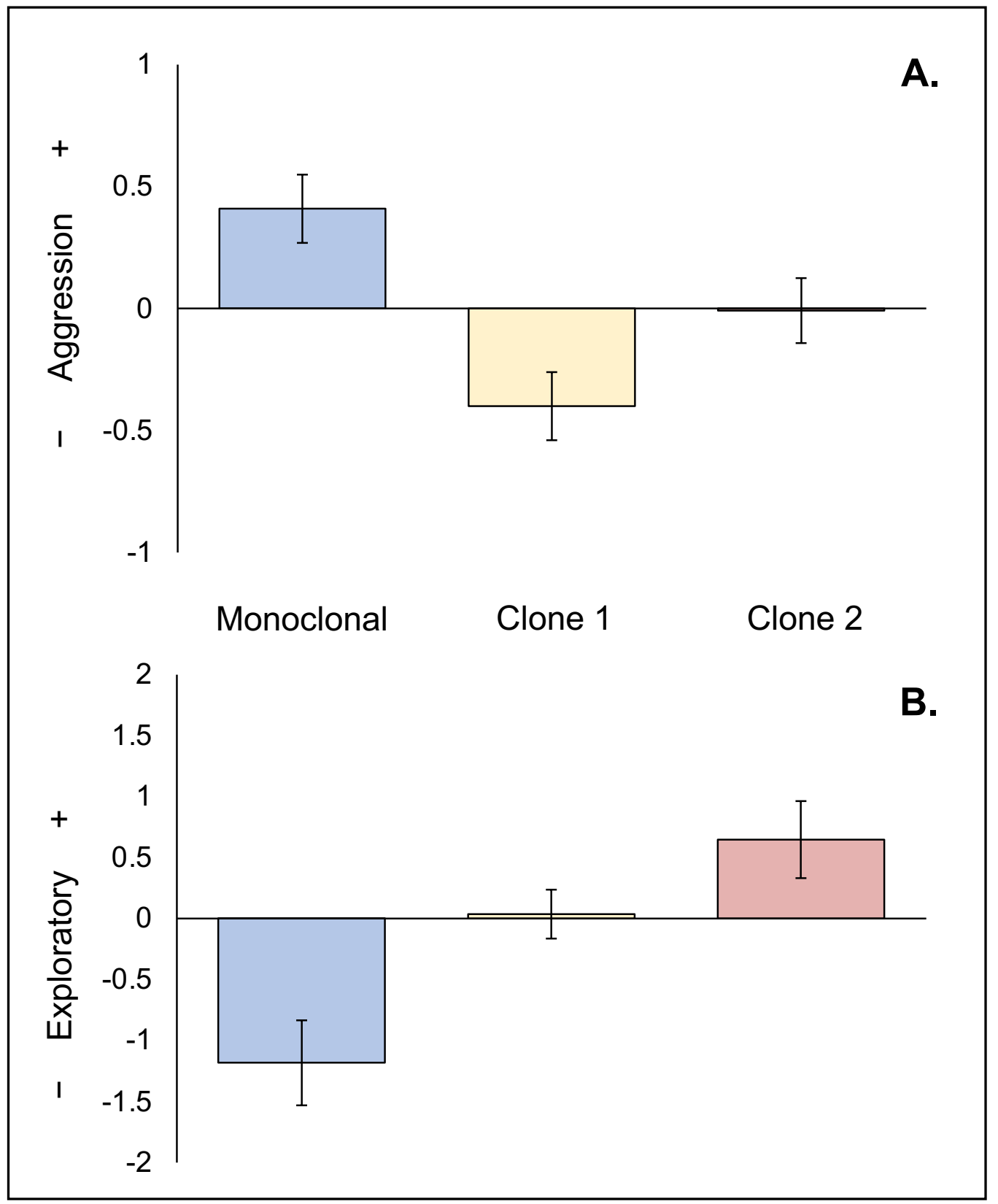


634 Figure 3: Visual representation of high intraclass correlation among individuals in the same

635 naïve-group trial. Within each treatment category (Monoclonal (blue), Clone 1 (yellow), Clone 2

636 (pink)) each row represents tracks of the three individuals in a single naïve-group trial. At each

637 time point (Pre-exposure (Pre), $4 \mathrm{wk}$, and $12 \mathrm{wk}$ ) the focal fish and two naïve partners were

638 tracked. For a given treatment, the same focal female was present at each time point, but her

639 two social partners were different individuals across time point.

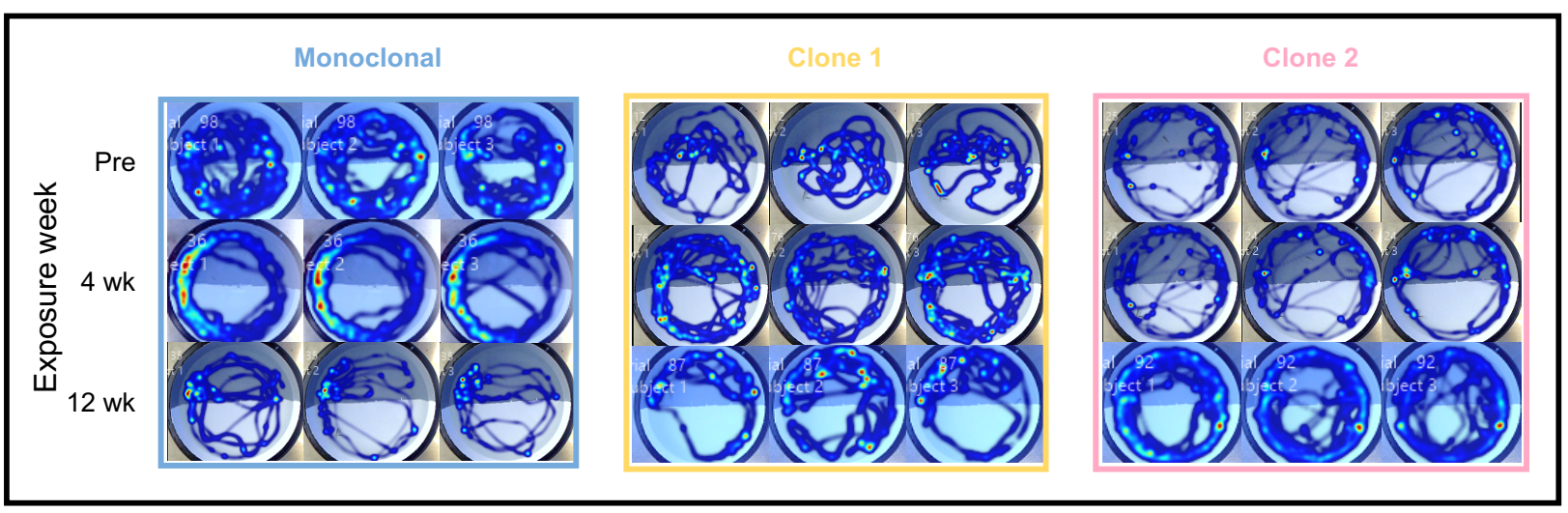

